Supporting Information Available

\title{
Synthesis of highly condensed polycyclic carbohydrates by reaction of a spirocyclic enamino sulfonate derived from D- xylofuranose with bifunctional reagents
}

\author{
Alessandra Cordeiro $^{a}$, María-Luisa Jimeno $^{b}$, Miguel Ángel Maestro ${ }^{c}$, María-José $^{2}$ \\ Camarasa $^{a}$, Ernesto Quesada ${ }^{a}$, and Ana San-Félix ${ }^{a *}$ \\ ${ }^{a}$ Instituto de Química Médica, CSIC, Juan de la Cierva 3, 28006 Madrid, Spain. ${ }^{b}$ Centro de Química \\ Orgánica "Manuel Lora-Tamayo", CSIC, Juan de la Cierva 3, 28006 Madrid, Spain. 'Departamento de \\ Química Fundamental, Facultade de Ciencias, Universidade da Coruña, 15071, La Coruña, Spain.
}

\section{Experimental section}

Table of contents

Index

General Methods

NMR procedures 
General methods. All reactions were carried out with magnetic stirring in oven-dried round bottomed flasks fitted with rubber septa or sealed tubes fitted with Teflon ${ }^{\circledR}$ caps unless otherwise noted.

Commercial reagents and solvents were used as received from the suppliers without further purification unless otherwise stated. Acetonitrile was dried prior to use by distillation from $\mathrm{CaH}_{2}$ and stored over Linde-type activated $4 \AA$ molecular sieves as described. ${ }^{1}$

Volatiles were removed by rotary evaporation under reduced pressure at $25-35{ }^{\circ} \mathrm{C}$. Analytical thin-layer chromatography (TLC) was performed on aluminium plates precoated with silica gel $60\left(\mathrm{~F}_{254}, 0.25 \mathrm{~mm}\right)$. Products were visualised from the TLC by exposure to ultraviolet light $(254 \mathrm{~nm})$ or by heating on a hot plate (aprox. $200{ }^{\circ} \mathrm{C}$ ), directly or after treatment with a $5 \%$ solution of phosphomolybdic acid or vanillin in ethanol. Separations were performed by preparative flash column chromatography ${ }^{2}$ on silica gel $60\left(\mathrm{PF}_{254}, 230-400 \mathrm{mesh}\right)$ or by preparative Centrifugal Circular Thin-Layer Chromatography (CCTLC) (Kiesegel $60 \mathrm{PF}_{254}$ gipshaltig, layer thickness of $1 \mathrm{~mm}$, flow rate $\left.4 \mathrm{~mL} \mathrm{~min}^{-1}\right)$.

Specific optical rotations were recorded using an automatic polarimeter with a cell of path length $1 \mathrm{dm}$. Mass spectra were registered in a quadrupole mass spectrometer with an electrospray source. Melting points were obtained on a hot plate apparatus and are uncorrected.

\footnotetext{
${ }^{1}$ Perrin, D. D.; Armarego, W. L. F. and Perrin D. R. in Purification of Laboratory Chemicals; 2nd Ed., Pergamon Press, Oxford. 1980.

${ }^{2}$ Still, W. C.; Kahn, M. and Mitra, A. J. Org. Chem. 1978, 43, 2923-2925.
} 
NMR procedures. Monodimensional ${ }^{1} \mathrm{H}$ and ${ }^{13} \mathrm{C}$ NMR spectra were obtained using standard conditions and were registered in $\mathrm{CDCl}_{3}$, acetone- $\mathrm{d}_{6}$, acetonitrile- $\mathrm{d}_{3}$, methanol$\mathrm{d}_{6}$ or DMSO- $\mathrm{d}_{6}$ as solvents with $-300,-400,-500$ NMR spectrometers. Solvents were used with higher deuteration degree than $99.5 \%$ and were filtered through a pad of neutral alumina in order to eliminate traces of water and acid prior to use. Chemical shifts for protons are reported in parts per million (ppm) downfield from tetramethylsilane referred to protonated residual peaks or specific signals due to deuterated solvents as internal references. Chemical shifts for proton-decoupled carbons are reported in parts per million ( $\mathrm{ppm}$ ) referenced to the deuterated solvents as internal standards. Multiplicities of NMR data are described by abbreviations: br (broad), s (singlet), d (doublet), t (triplet), q (quadruplet), m (complex multiplet). Data are reported using the following convention: chemical shift (multiplicity, integration and coupling constant).

Carbon and proton assignments were based on DEPT, HSQC, HMQC, HMBC, gradient-COSY and NOE-differential experiments. Homonuclear 2D spectra were acquired in the phase-sensitive mode. Data were collected in a 1024 x 256 matrix with a spectral width of $5000 \mathrm{~Hz}$ and a 2 s recycle delay and processed in a 1024 x 1024 matrix. 2D Inverse proton detected heteronuclear shift correlation spectra, gHSQC and gHMBC, were obtained with the following conditions: data were collected in a $4096 \mathrm{x}$ 128 matrix with a spectral width of $8000 \mathrm{~Hz}$ in the proton domain and $25000 \mathrm{~Hz}$ in the carbon domain, and processed in a 4096 x 512 matrix. The gHSQC experiment was optimized for one bond heteronuclear coupling constant of $145 \mathrm{~Hz}$. The gHMBC experiment was optimized for long range coupling constants of $8 \mathrm{~Hz}$. 
List of abbreviations

$\mathrm{CH}_{2} \mathrm{Cl}_{2}$

CCTLC

ESI

$\mathrm{Hz}$

J

$\mathrm{mL}$

$\mu \mathrm{L}$

mmol

NMR

ppm
Dichloromethane

Circular Centrifugal Thin Layer

Chromatography

Electrospray ionization

Hertz

Coupling constant

Milliliter

Microliter

Millimol

Nuclear Magnetic Resonance

Parts per million 
${ }^{1} \mathrm{H}-\mathrm{NMR}\left[500 \mathrm{MHz},\left(\mathrm{CD}_{3}\right)_{2} \mathrm{CO}\right]$

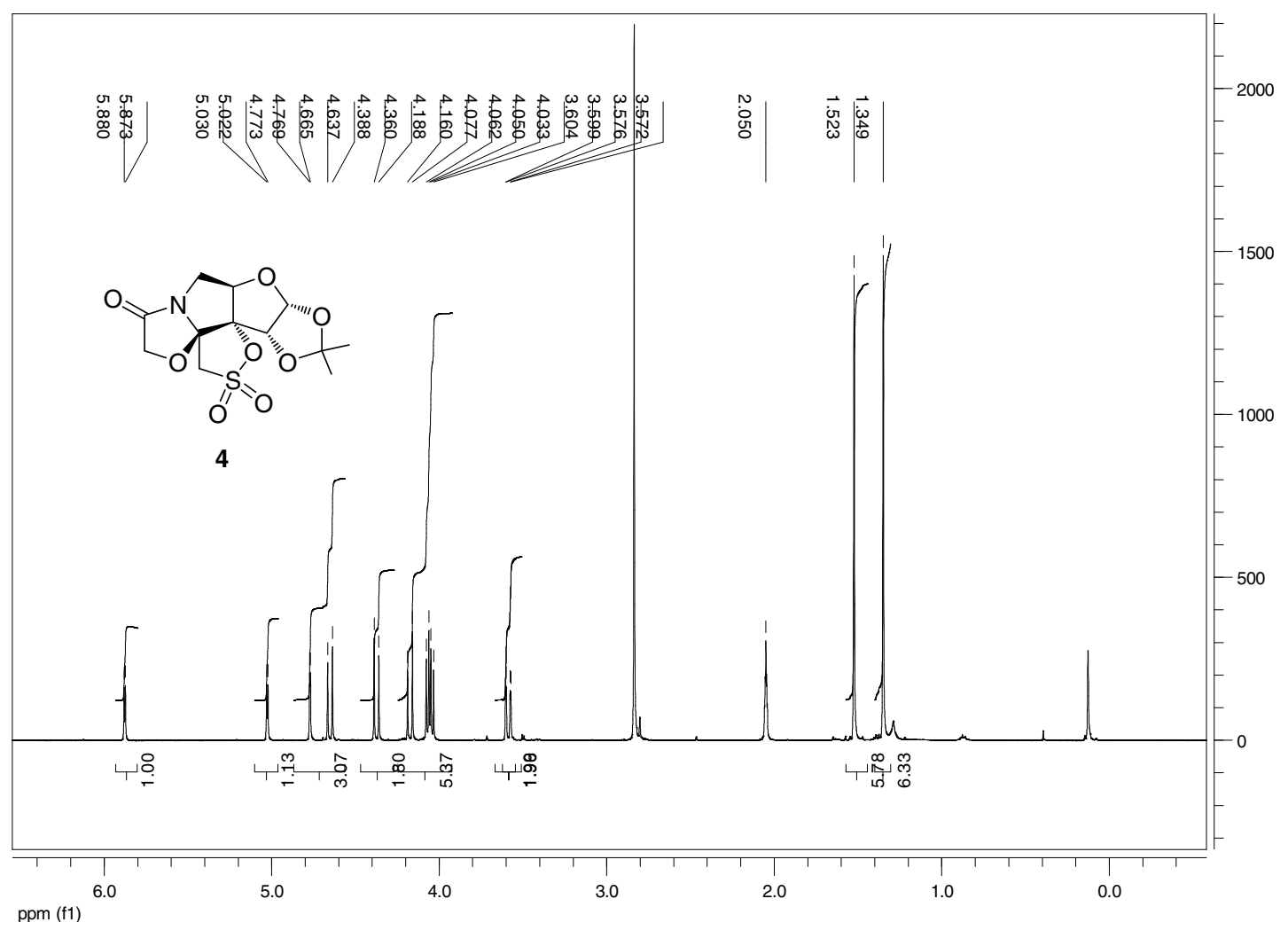

${ }^{13} \mathrm{C}-\mathrm{NMR}\left[75 \mathrm{MHz},\left(\mathrm{CD}_{3}\right)_{2} \mathrm{CO}\right]$

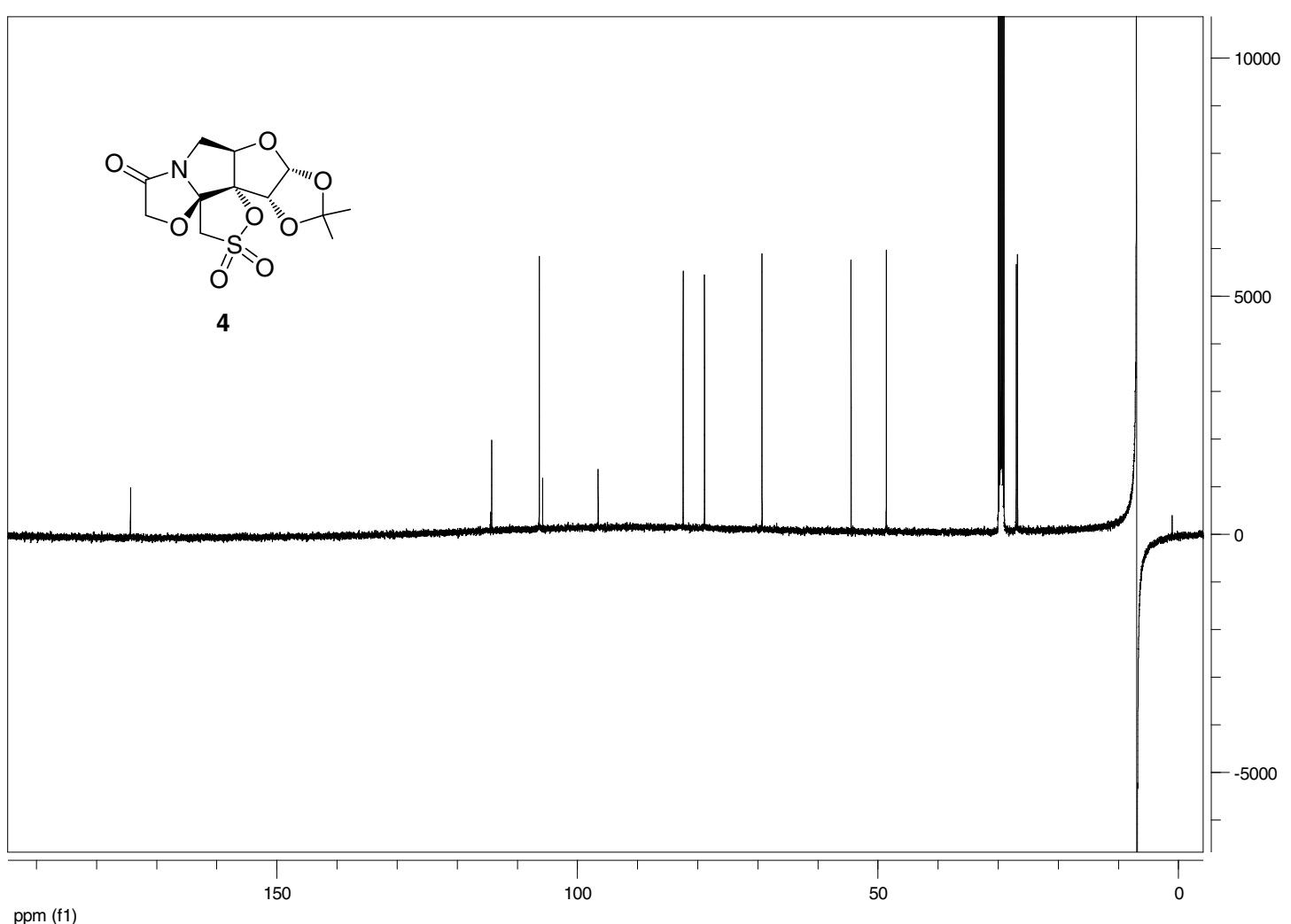


${ }^{1} \mathrm{H}-\mathrm{NMR}\left[300 \mathrm{MHz},\left(\mathrm{CD}_{3}\right)_{2} \mathrm{CO}\right]$

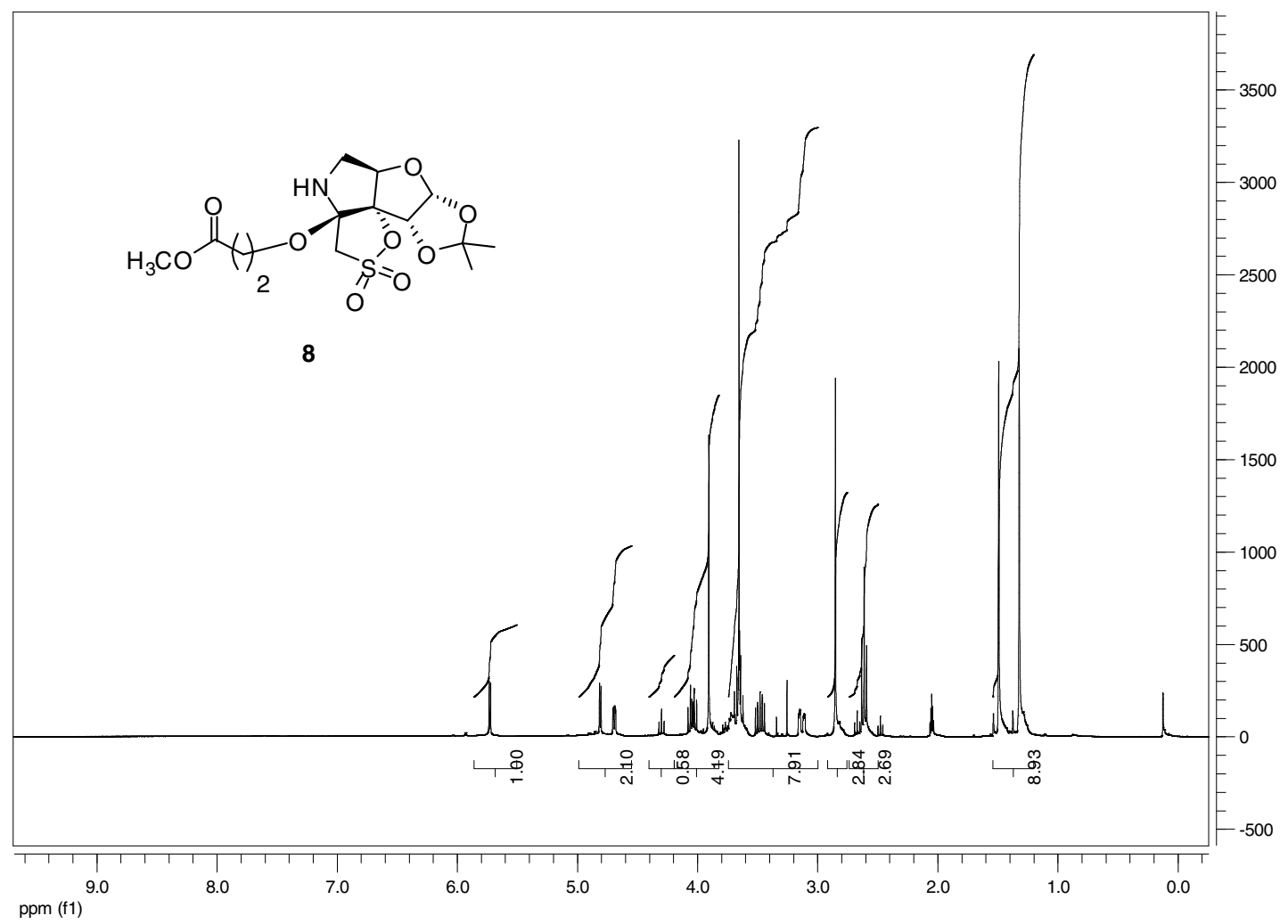

${ }^{13} \mathrm{C}-\mathrm{NMR}\left[100 \mathrm{MHz},\left(\mathrm{CD}_{3}\right)_{2} \mathrm{CO}\right]$

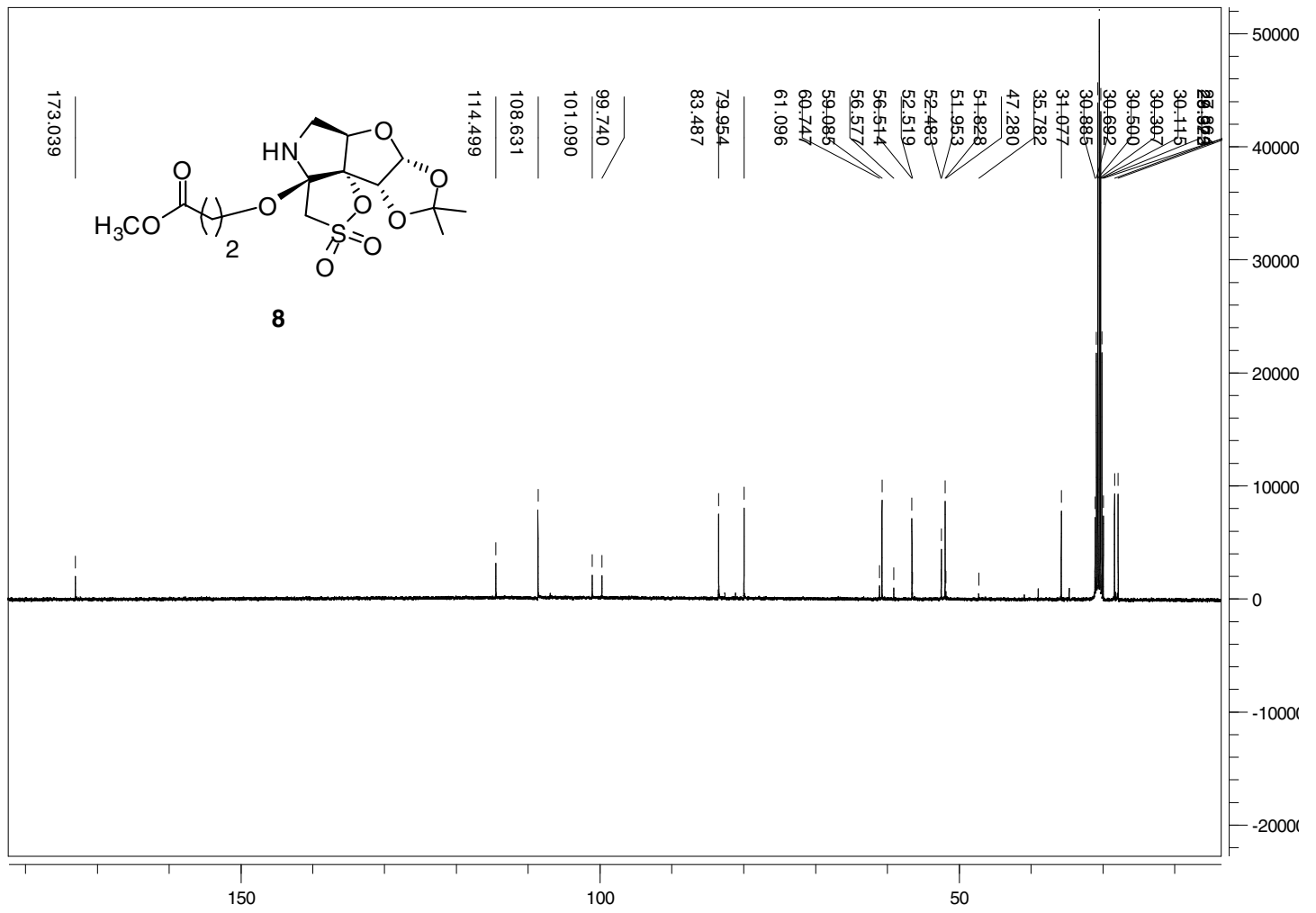

ppm (f1) 
${ }^{1} \mathrm{H}-\mathrm{NMR}\left[300 \mathrm{MHz},\left(\mathrm{CD}_{3}\right)_{2} \mathrm{CO}\right]$

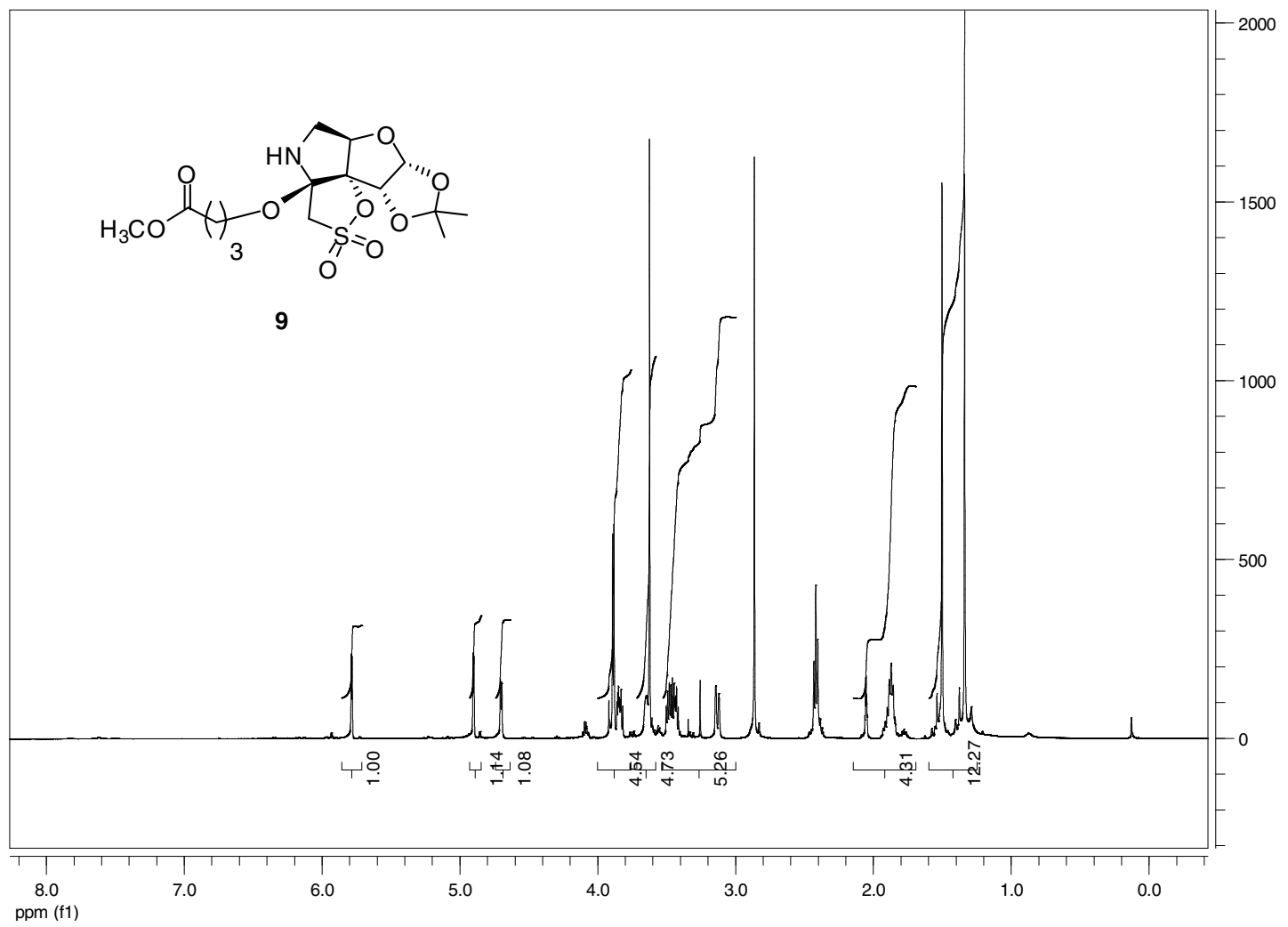

${ }^{13} \mathrm{C}-\mathrm{NMR}\left[100 \mathrm{MHz},\left(\mathrm{CD}_{3}\right)_{2} \mathrm{CO}\right]$

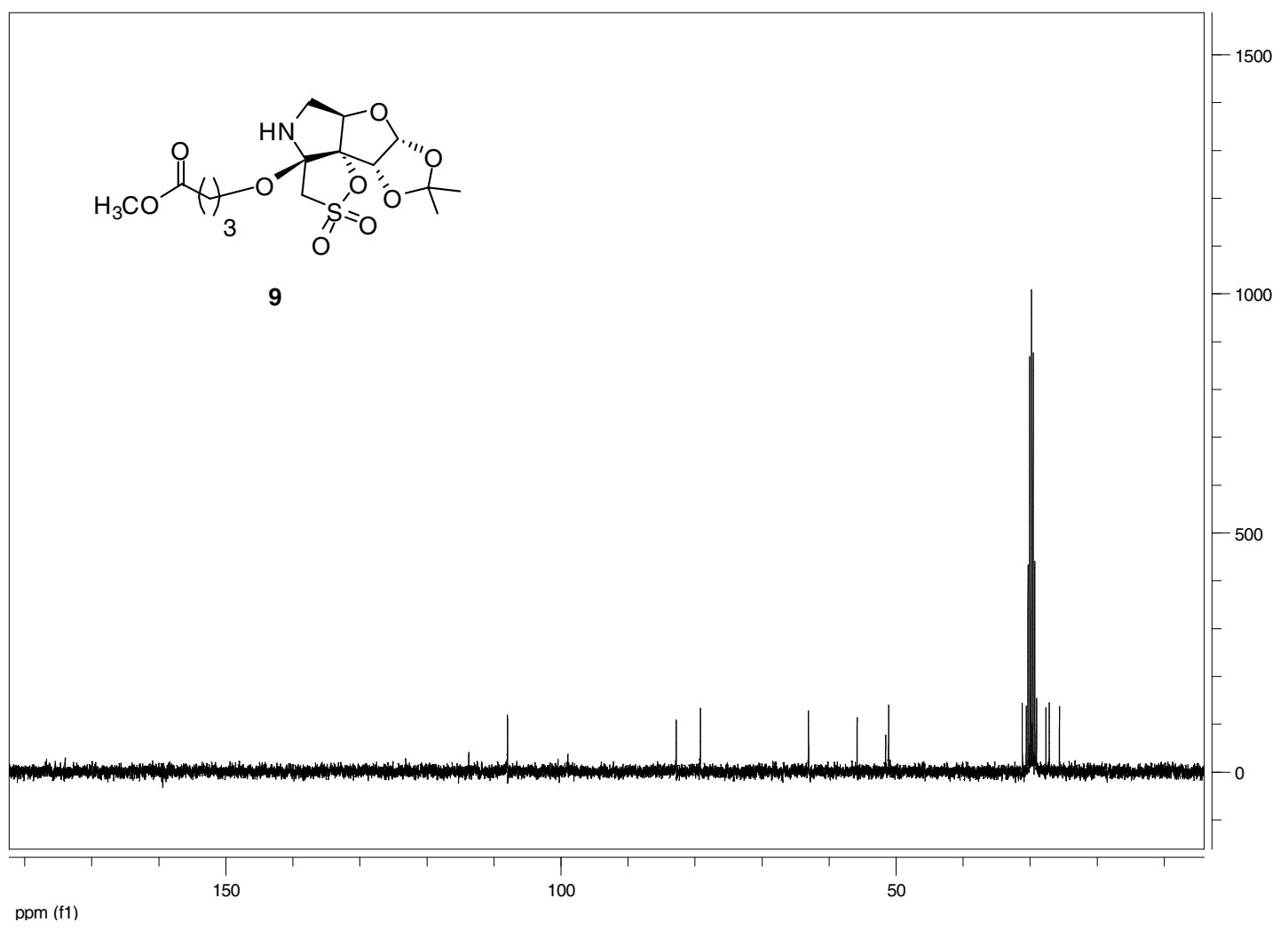


${ }^{1} \mathrm{H}-\mathrm{NMR}\left[300 \mathrm{MHz},\left(\mathrm{CD}_{3}\right)_{2} \mathrm{CO}\right]$

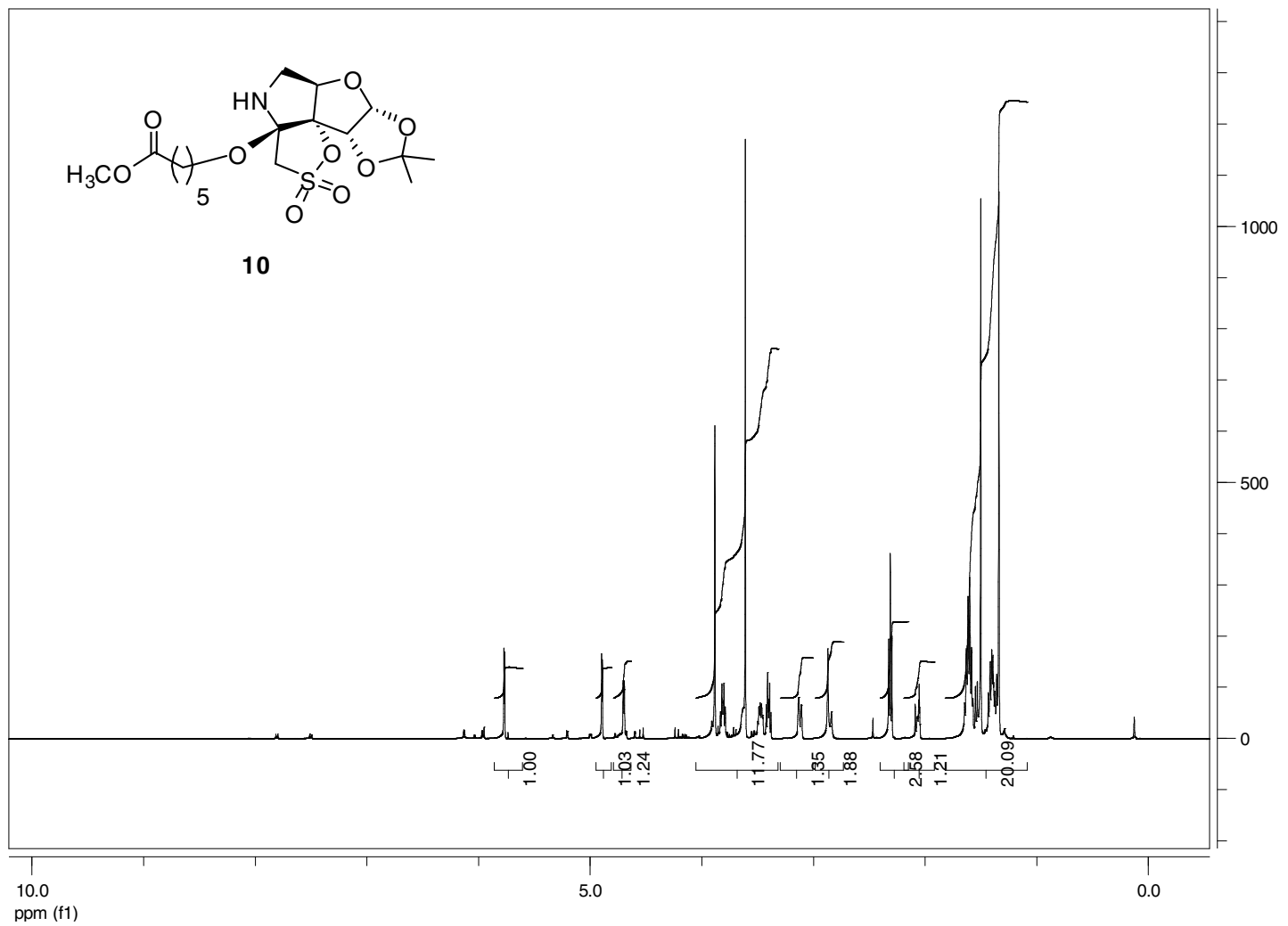

${ }^{13} \mathrm{C}-\mathrm{NMR}\left[100 \mathrm{MHz},\left(\mathrm{CD}_{3}\right)_{2} \mathrm{CO}\right]$

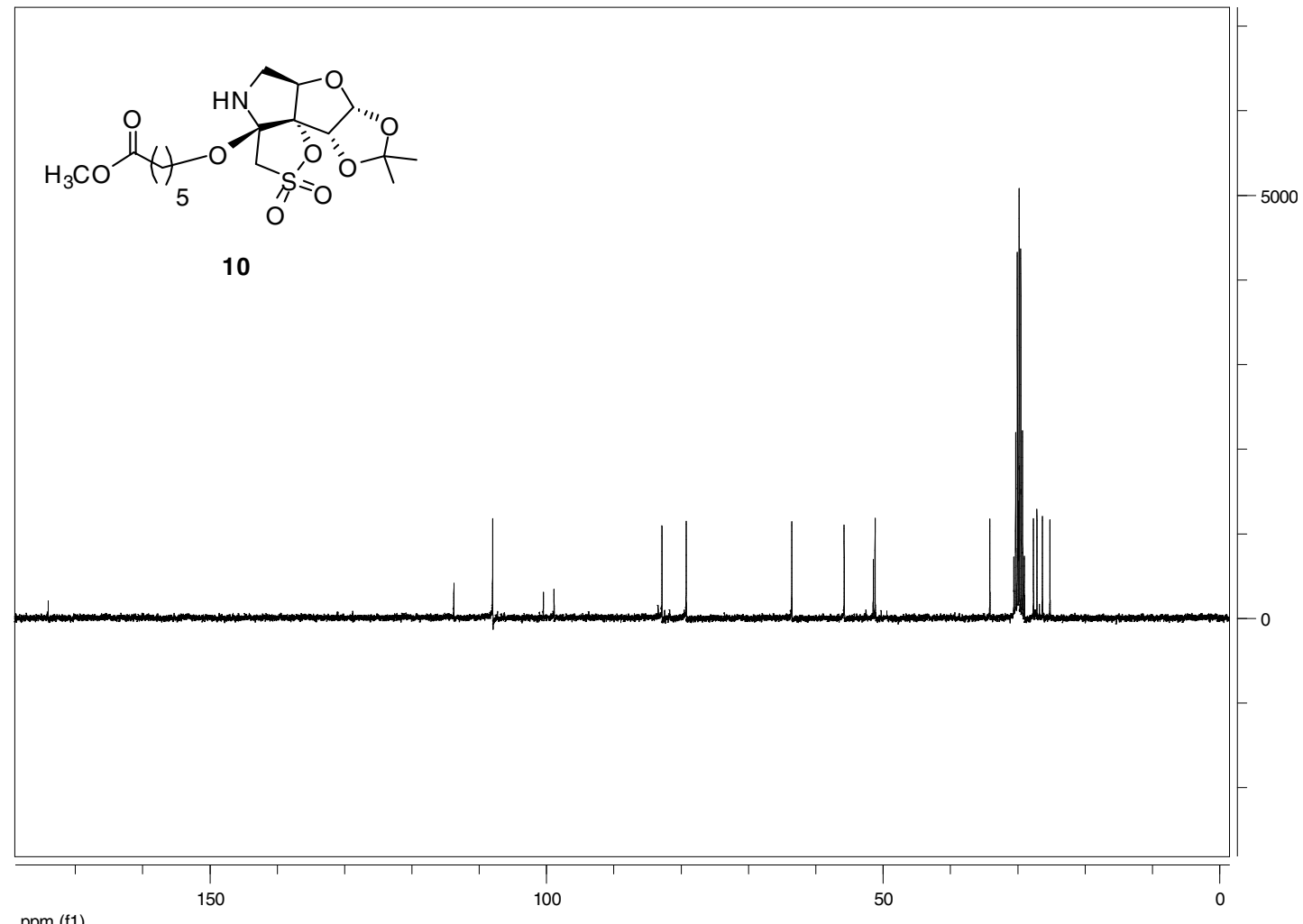


${ }^{1} \mathrm{H}-\mathrm{NMR}\left[300 \mathrm{MHz},\left(\mathrm{CD}_{3}\right)_{2} \mathrm{CO}\right]$

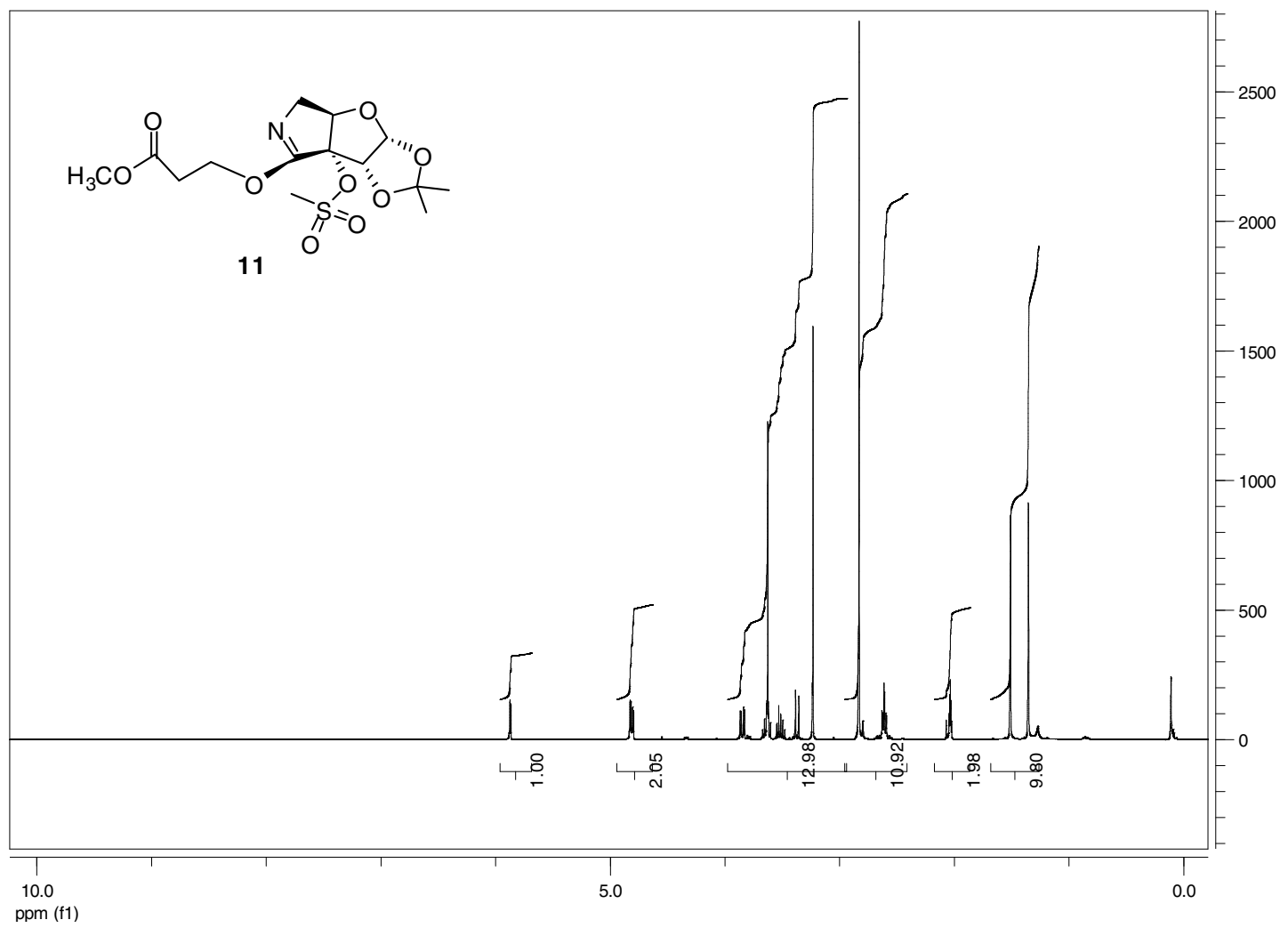

${ }^{13} \mathrm{C}-\mathrm{NMR}\left[75 \mathrm{MHz},\left(\mathrm{CD}_{3}\right)_{2} \mathrm{CO}\right]$

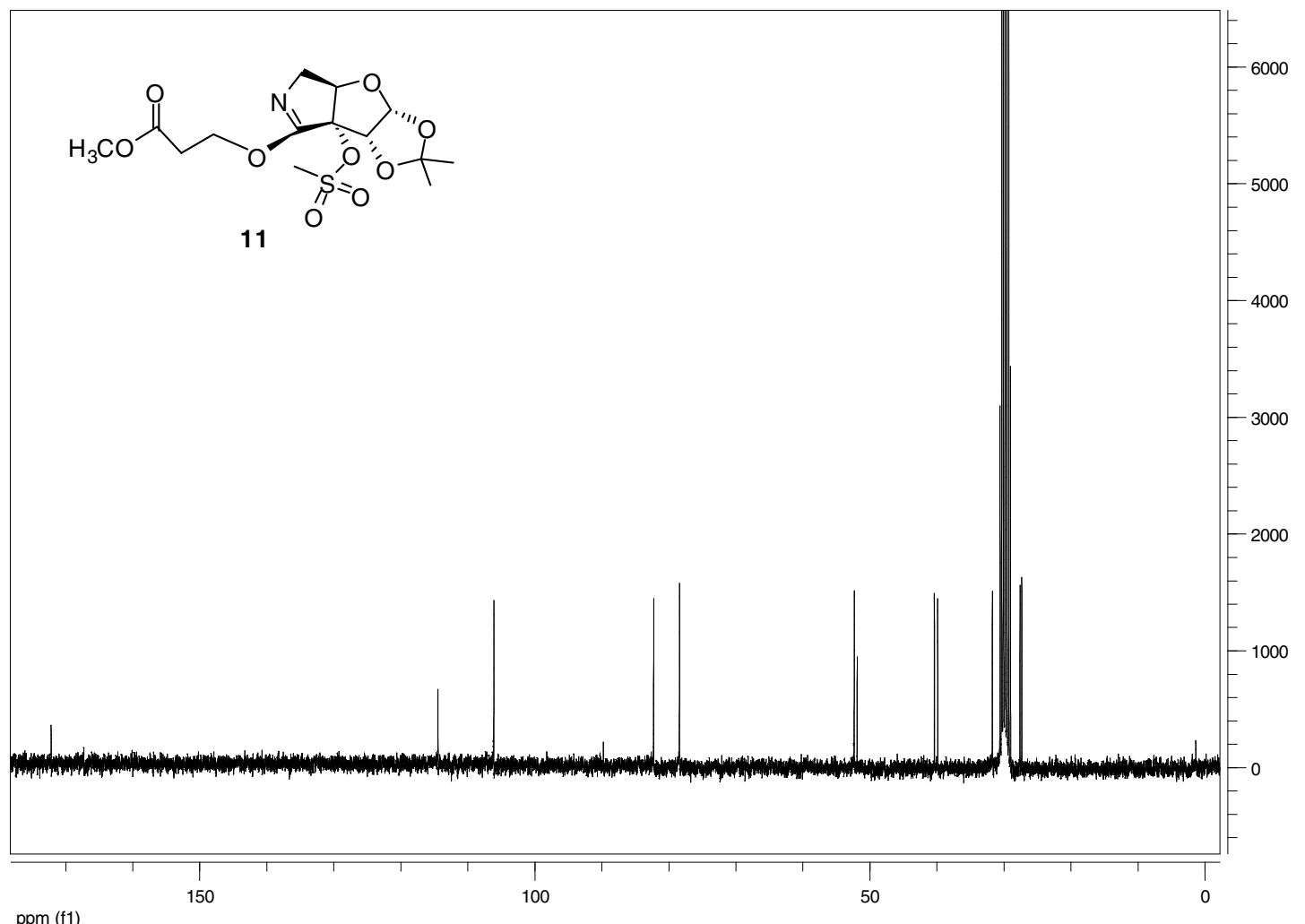


${ }^{1} \mathrm{H}-\mathrm{NMR}\left[300 \mathrm{MHz},\left(\mathrm{CD}_{3}\right)_{2} \mathrm{CO}\right]$

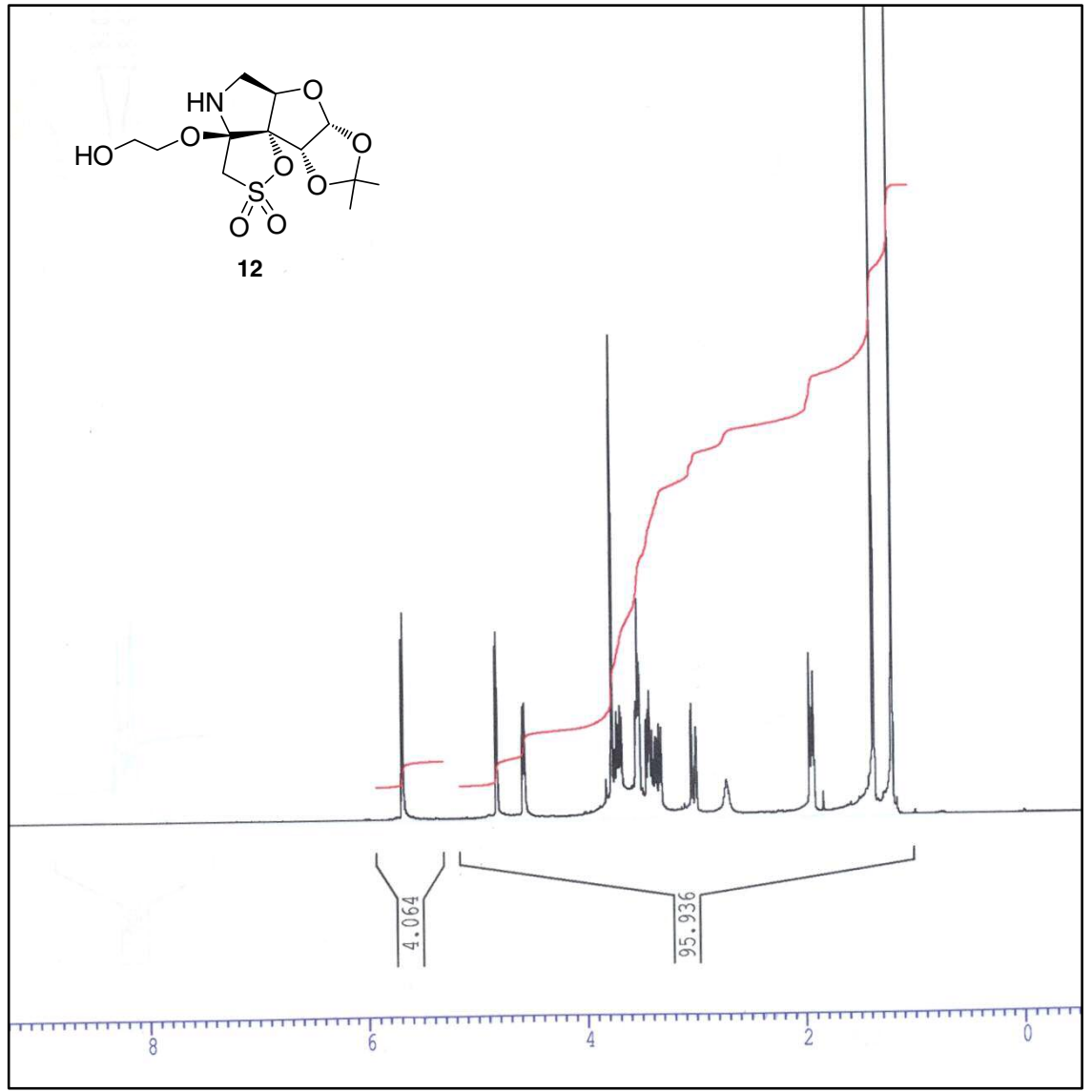

${ }^{13} \mathrm{C}-\mathrm{NMR}\left[75 \mathrm{MHz},\left(\mathrm{CD}_{3}\right)_{2} \mathrm{CO}\right]$

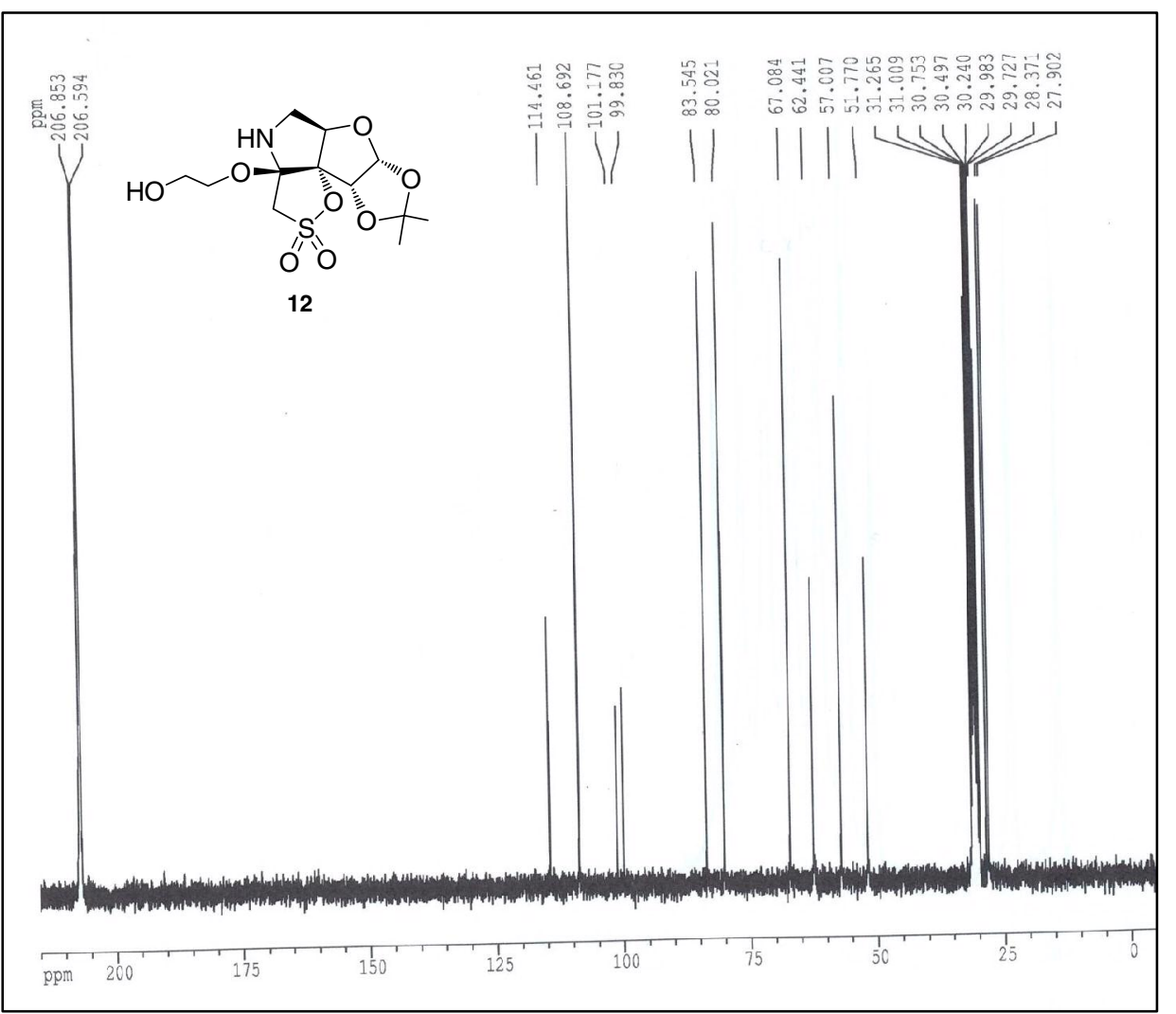


${ }^{1} \mathrm{H}-\mathrm{NMR}\left[300 \mathrm{MHz},\left(\mathrm{CD}_{3}\right)_{2} \mathrm{CO}\right]$

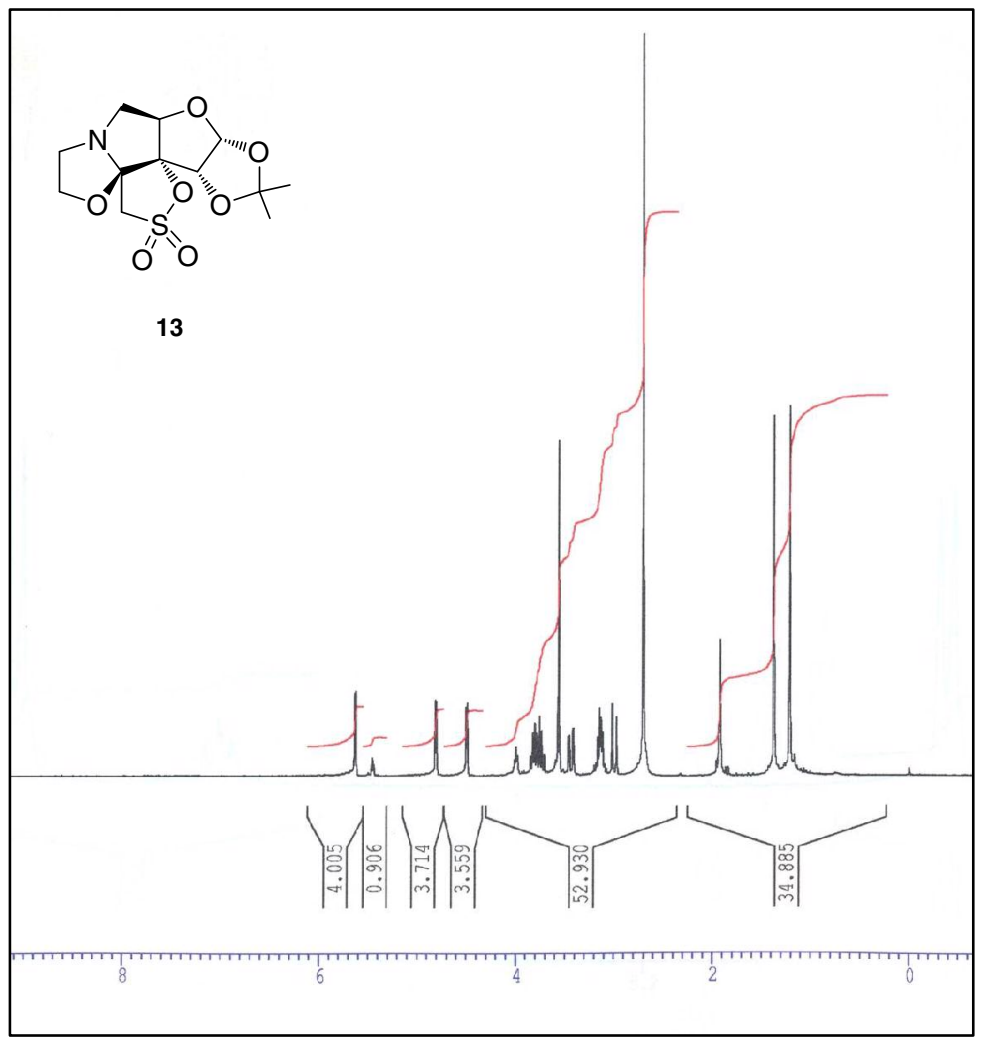

${ }^{13} \mathrm{C}-\mathrm{NMR}\left[75 \mathrm{MHz},\left(\mathrm{CD}_{3}\right)_{2} \mathrm{CO}\right]$

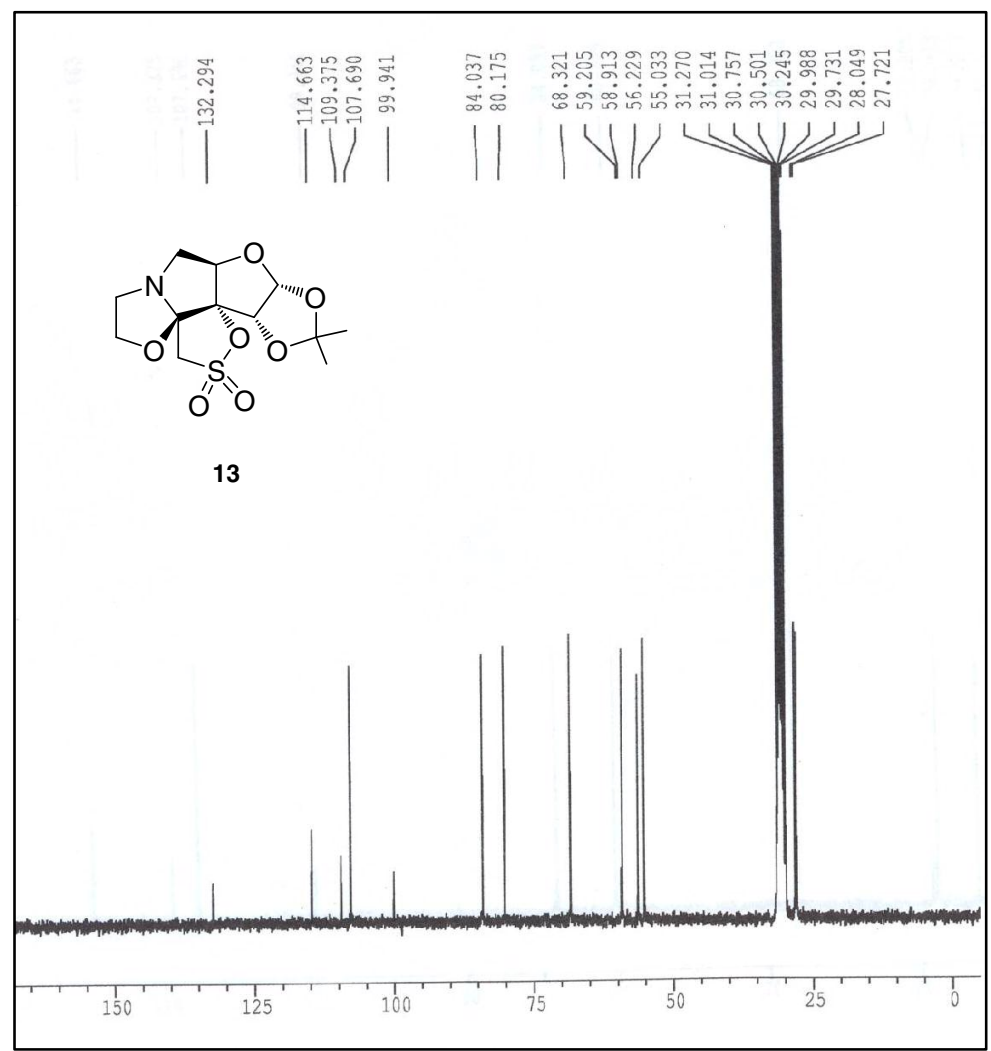


${ }^{1} \mathrm{H}-\mathrm{NMR}\left[300 \mathrm{MHz},\left(\mathrm{CD}_{3}\right)_{2} \mathrm{CO}\right]$

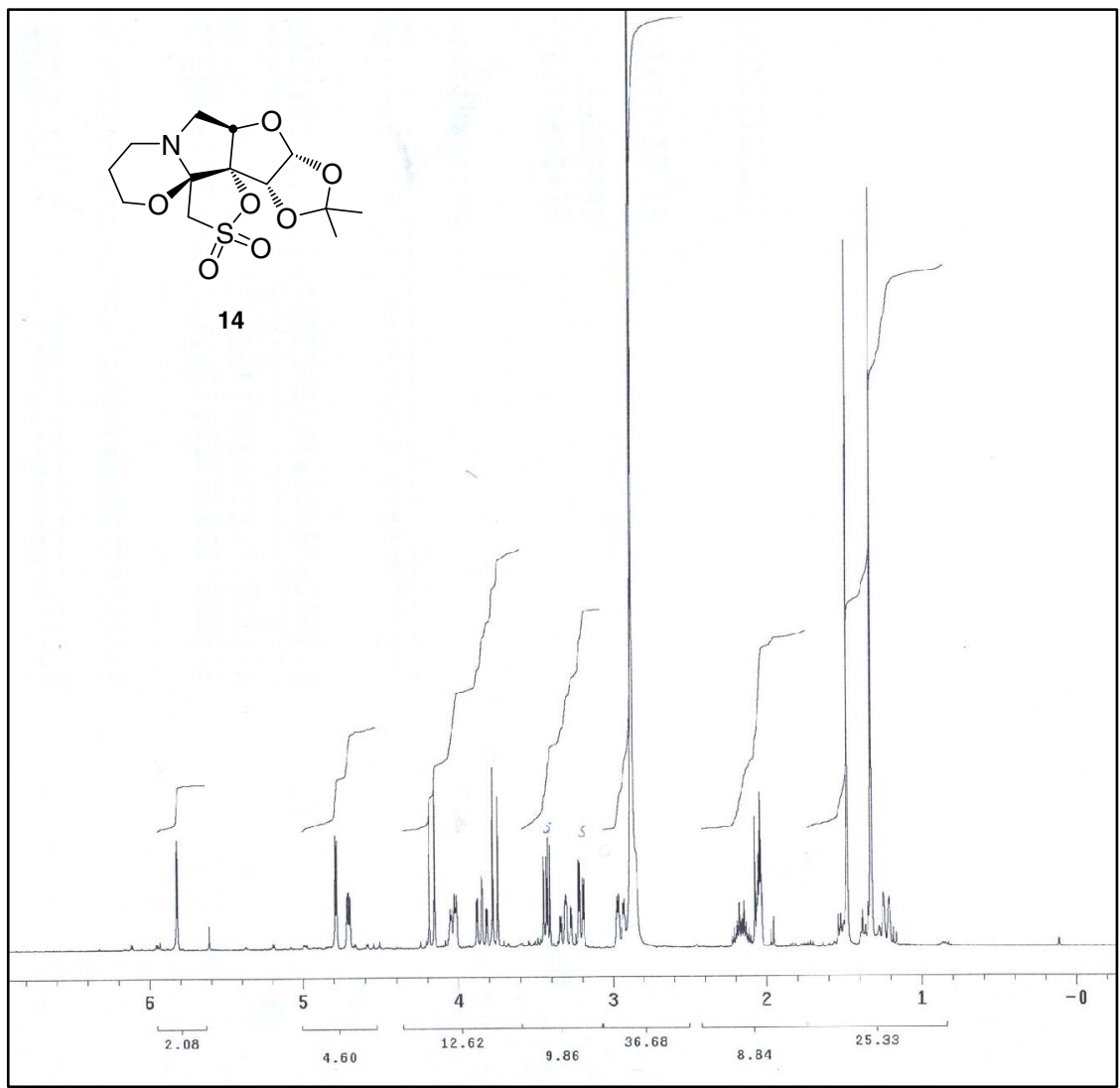

${ }^{13} \mathrm{C}-\mathrm{NMR}$ [75 MHz, $\left(\mathrm{CD}_{3}\right)_{2} \mathrm{CO}$ ]

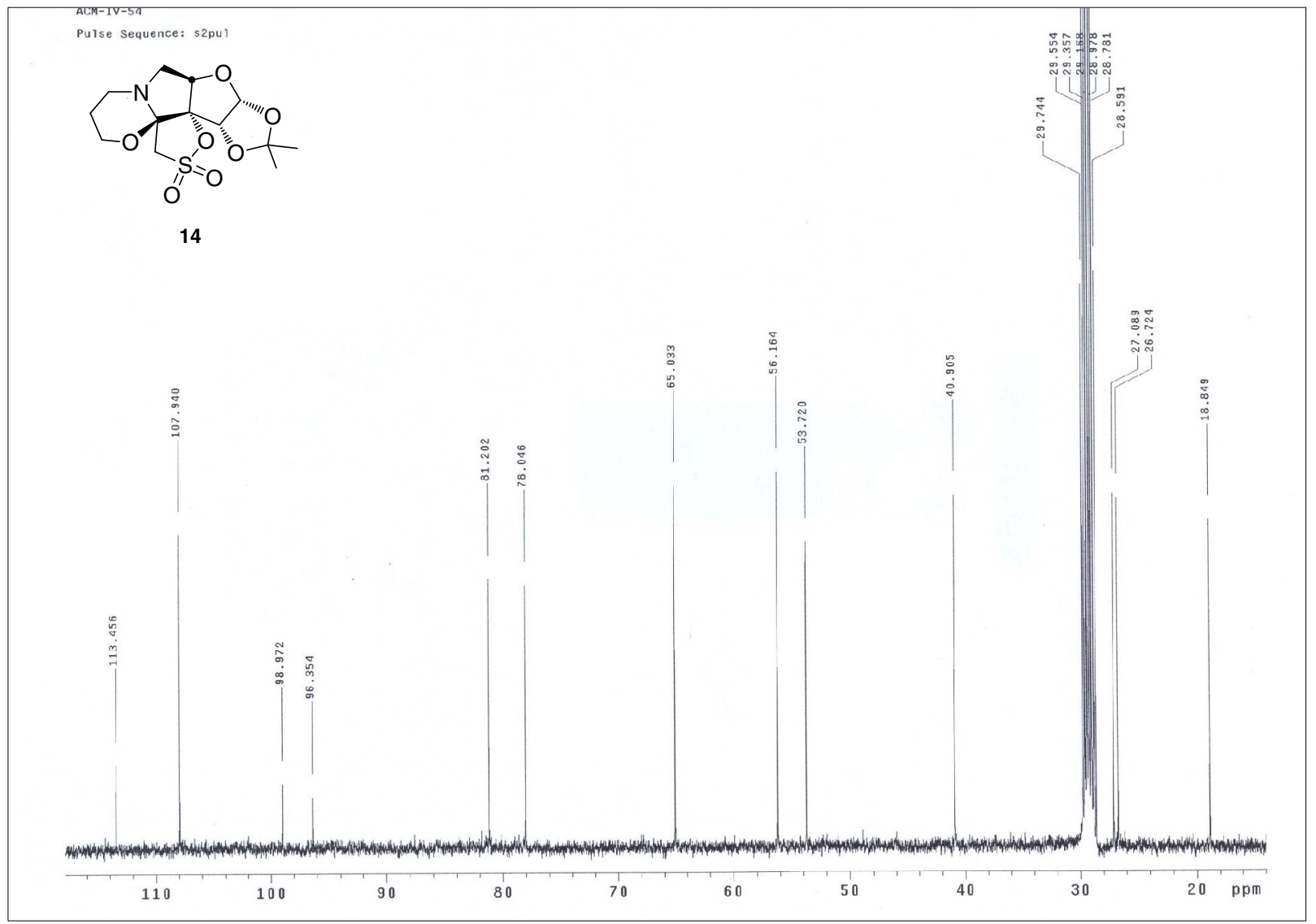


${ }^{1} \mathrm{H}-\mathrm{NMR}\left[300 \mathrm{MHz},\left(\mathrm{CD}_{3}\right)_{2} \mathrm{CO}\right]$

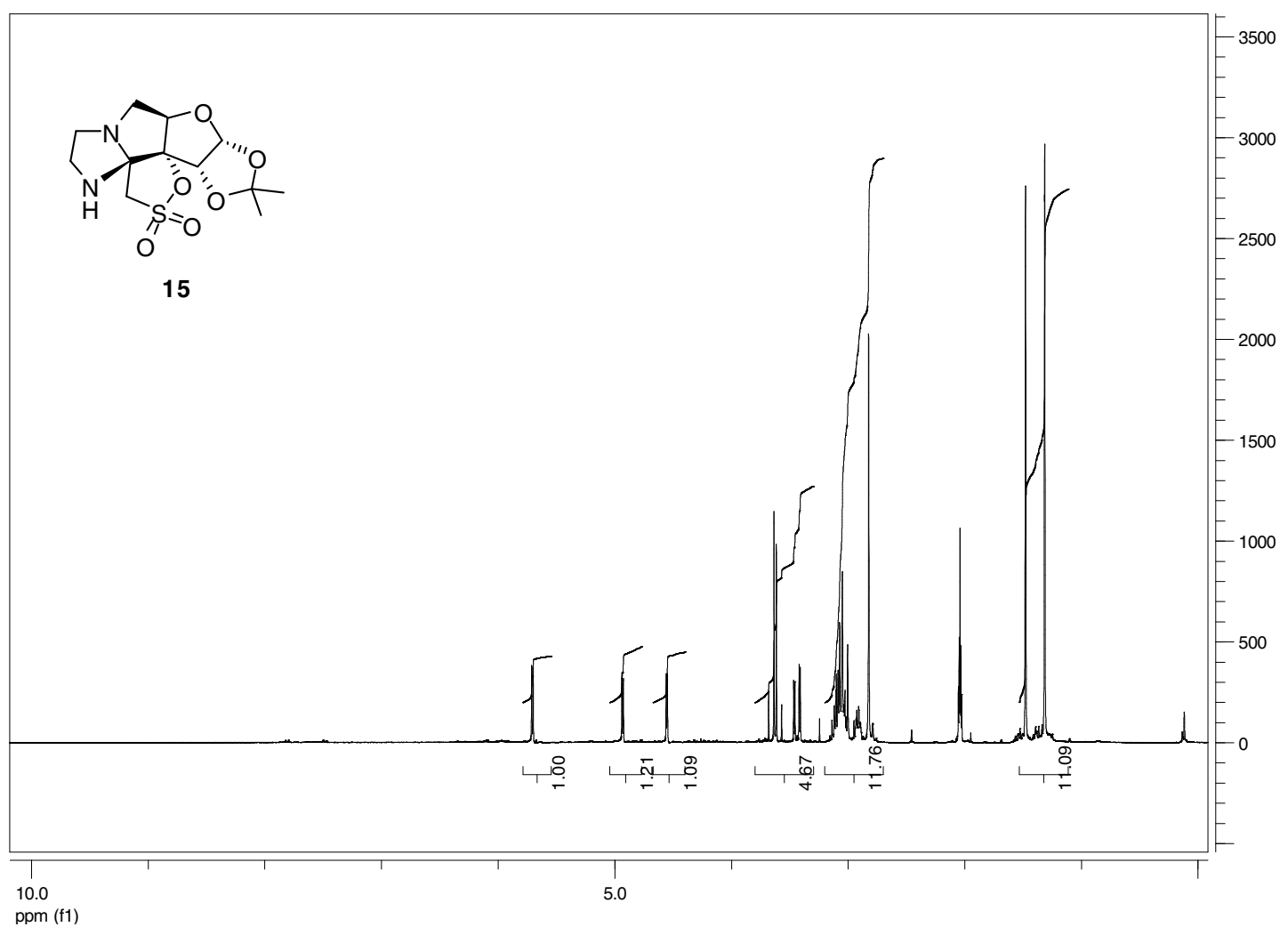

${ }^{13} \mathrm{C}-\mathrm{NMR}\left[75 \mathrm{MHz},\left(\mathrm{CD}_{3}\right)_{2} \mathrm{CO}\right]$

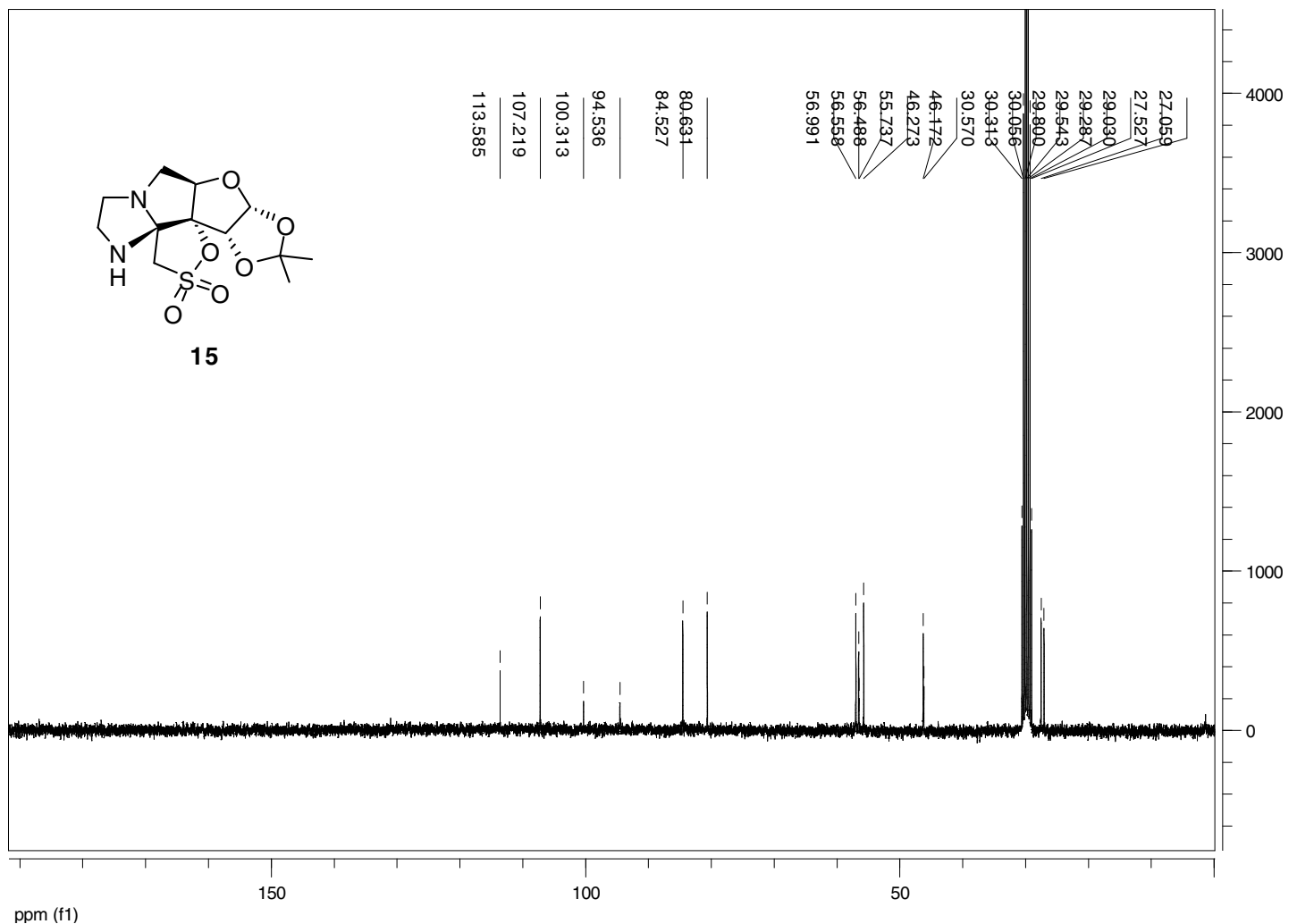


${ }^{1} \mathrm{H}-\mathrm{NMR}\left[300 \mathrm{MHz},\left(\mathrm{CD}_{3}\right)_{2} \mathrm{CO}\right]$

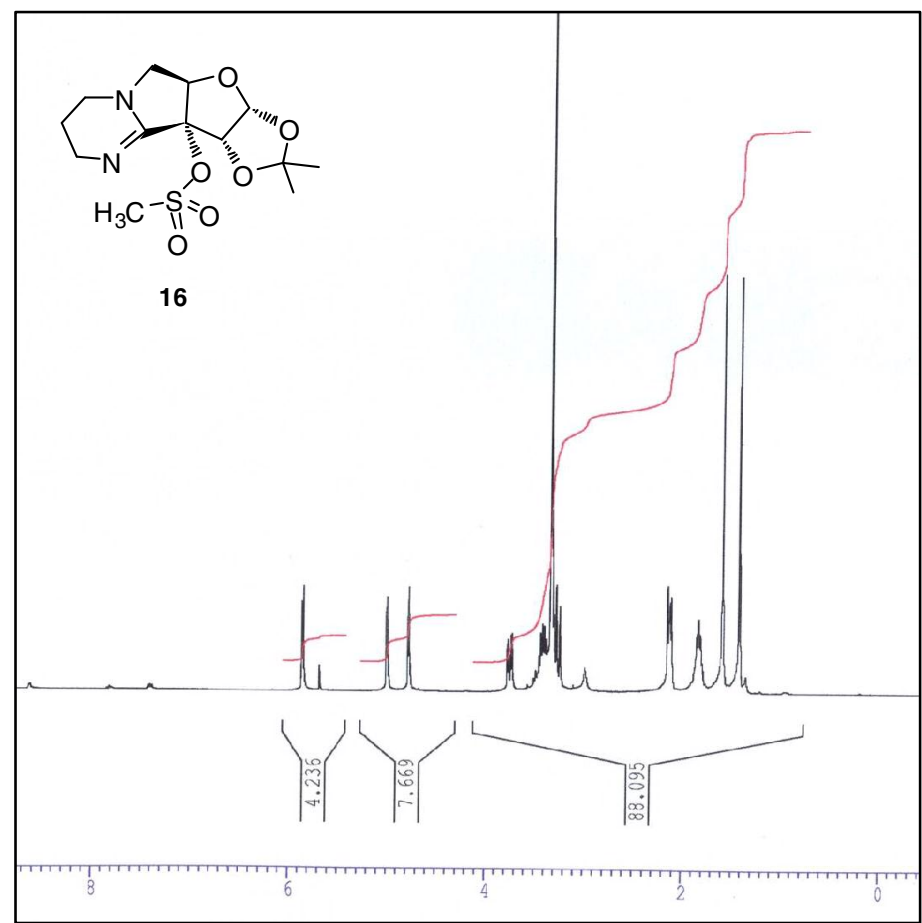

${ }^{13} \mathrm{C}-\mathrm{NMR}\left[75 \mathrm{MHz},\left(\mathrm{CD}_{3}\right)_{2} \mathrm{CO}\right]$

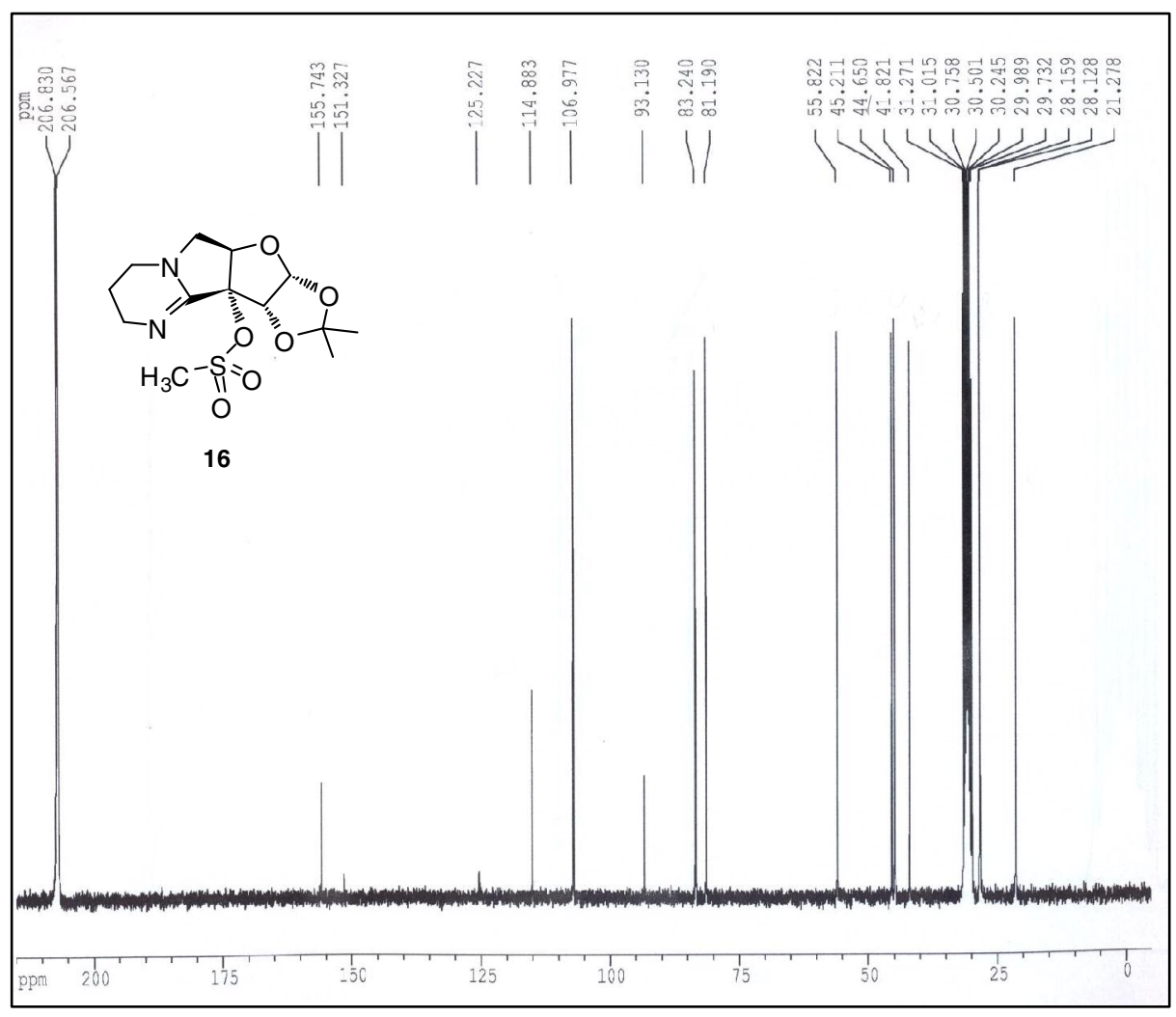


${ }^{1} \mathrm{H}-\mathrm{NMR}\left[400 \mathrm{MHz},\left(\mathrm{CD}_{3}\right)_{2} \mathrm{CO}\right]$

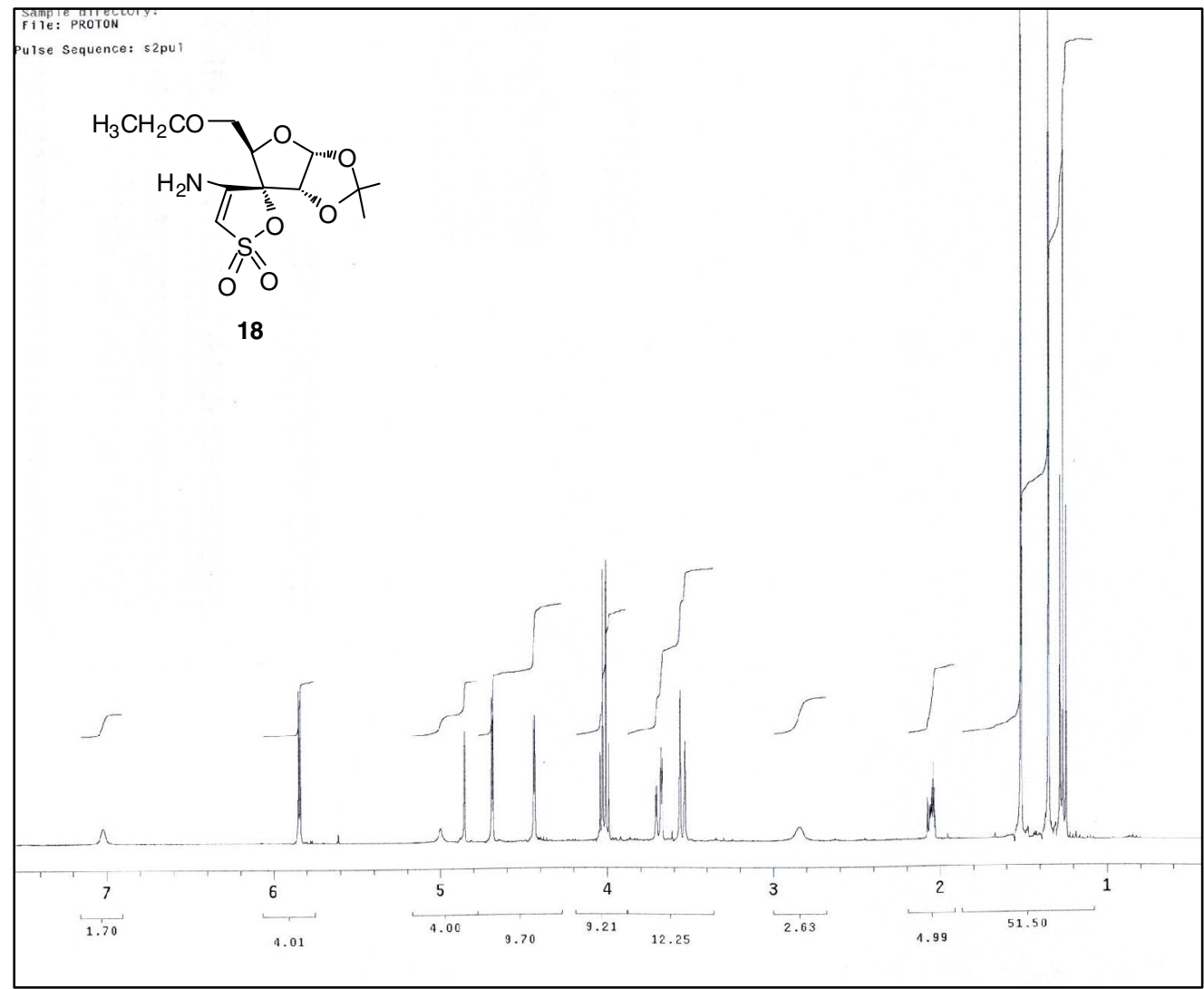

${ }^{13} \mathrm{C}-\mathrm{NMR}\left[100 \mathrm{MHz},\left(\mathrm{CD}_{3}\right)_{2} \mathrm{CO}\right]$

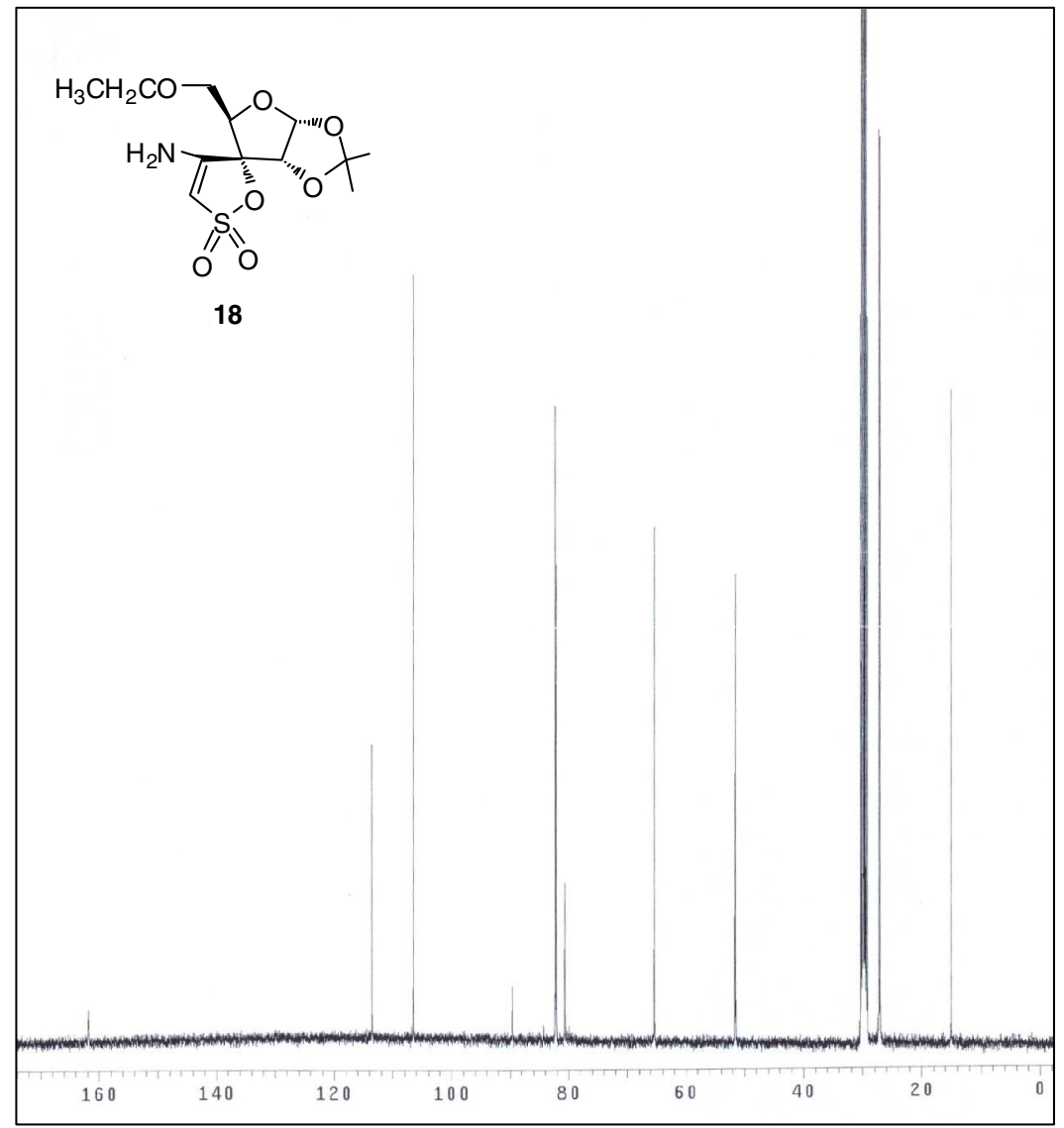


